\title{
Electrical stimulation versus kinesitherapy in improving functional fitness in older women: A randomized controlled trial
}

\author{
Martina Anna Maggioni ${ }^{\mathrm{a}, *}$, Emiliano Cè ${ }^{\mathrm{a}, \mathrm{b}}$, Susanna Rampichini ${ }^{\mathrm{a}}$, Marco Ferrario ${ }^{\mathrm{c}}$, \\ Geremia Giordano ${ }^{c}$, Arsenio Veicsteinas ${ }^{\mathrm{a}, \mathrm{b}}$, Giampiero Merati ${ }^{\mathrm{a}, \mathrm{b}}$ \\ ${ }^{a}$ Department of Sport Sciences, Nutrition and Health, University of Milan, via G. Colombo 71, I-20133 Milan, Italy \\ ${ }^{\mathrm{b}}$ Sport Medicine Centre, Don C. Gnocchi Foundation, via Capecelatro 66, I-20148 Milan, Italy \\ ${ }^{\mathrm{c}}$ Rehabilitation Unit, Palazzolo Institute, Don C. Gnocchi Foundation, via L. Palazzolo 21, I-20149 Milan, Italy
}

\section{A R T I C L E I N F O}

\section{Article history:}

Received 10 December 2008

Received in revised form 24 April 2009

Accepted 28 April 2009

Available online 28 May 2009

\section{Keywords:}

Elderly

Exercise

Rehabilitation

Physical fitness

\begin{abstract}
A B S T R A C T
Long-stay hospitalized elderly patients frequently develop a decrease in strength, cardiovascular fitness and balance, which can be improved by exercise. Neuromuscular electrical stimulation (ES) might also be effective in this population. The effect of different lower limb rehabilitation programs (3 interventions/week for 6 weeks) on functional fitness in 40 hospitalized old females (82 \pm 7 years) were evaluated: kinesitherapy (KT), ES, KT alternated with ES (KT + ES), and no treatment (C). Outcome variables after rehabilitation were: (a) maximal strength of leg extensor and finger flexor muscles; (b) cardiorespiratory fitness (6-min walking test, 6MWT; heart rate, HR); (c) static and dynamic balance and gait skills (Tinetti test). The maximal strength of leg extensor (but not finger flexor) muscles significantly improved in ES (+26\%) and KT + ES (+16\%) groups only. The distance covered during the 6MWT significantly increased in $\mathrm{KT}(+15 \%)$, ES $(+14 \%)$ and $\mathrm{KT}+\mathrm{ES}(+9 \%)$ groups, but the post-test HR recovery improved in KT group only. Balance (but not gait) skill scores significantly and similarly increased in KT (+11\%), ES (+10\%) and $\mathrm{KT}+\mathrm{ES}(+11 \%)$ groups. In long-stay hospitalized old females ES and KT + ES improved lower limbs isometric strength. Walking ability was improved by all treatments, whereas cardiovascular performance and recovery were ameliorated by KT only.
\end{abstract}

(c) 2009 Elsevier Ireland Ltd. All rights reserved.

\section{Introduction}

Long-stay hospitalized old patients frequently develop a rapid decrease in muscular strength, cardiovascular fitness and balance, as reported in many observational studies (Gillick et al., 1982; Warshaw et al., 1982; Hirsch et al., 1990; Inouye et al., 1993). Such a decline is usually a consequence of limited physical activity (Gillick et al., 1982; Creditor, 1993; Inouye et al., 1993) and occurs early, within a few days after the admission (Lazarus et al., 1991), regardless of the underlying pathology. Therefore, interest is growing in investigating the possible interventions, such as physical exercise, which could limit musculoskeletal and cardiopulmonary decline in long-stay hospitalized adults (Vuori, 1995). This aspect is particularly relevant in women, as they are at greater risk of motor impairment, due to a sex-related decline in muscle strength (Rantanen et al., 1994). It is therefore important for

\footnotetext{
* Corresponding author. Tel.: +39 025031 4639; fax: +39 0250314639

E-mail address: martina.maggioni@unimi.it (M.A. Maggioni).
}

hospitalized elderly women to exercise regularly to maintain and/ or recover their functional fitness.

Many studies showed that an exercise intervention improves functional fitness in hospitalized older women (Nichols et al., 1995; Nakamura et al., 2007). A protocol consisting of isometric and isotonic voluntary contractions of upper limbs, trunk and lower limb muscles was generally adopted in these studies.

Neuromuscular electrical stimulation has also been successfully employed in many rehabilitative programs in the last decades. ES increases muscle strength, induces changes in muscle fiber composition and capillary system structure, prevents muscle atrophy due to the prolonged immobilization (Mysiw and Jackson, 2000), decreases pain (Durmus et al., 2007) and increases functional fitness (Mysiw and Jackson, 2000; Pekindil et al., 2001). Moreover, ES seems to be particularly indicated in patients with difficulty or contraindications to perform a voluntary exercise program, e.g. after surgery, or in subjects who are not familiar with active, voluntary exercise (Mysiw and Jackson, 2000). Finally, ES was recently shown to be an effective rehabilitative approach in patients with complex pathologies, such as chronic obstructive pulmonary disease 
(Neder et al., 2002) and congestive heart failure (Quittan et al., 2001). In these conditions the purpose of ES is not only to increase muscle strength, but also to reduce effort during daily activity and to consequently improve self-sufficiency.

However, only few studies so far focused on the different effects of voluntary contraction exercise versus ES on functional fitness in hospitalized elderly patients. In addition, most studies targeted only specific pathologies, e.g. rheumatoid arthritis, osteoarthritis, etc. (Durmus et al., 2007; Piva et al., 2007). Finally, no studies addressed the question whether the effects of the two rehabilitative procedures could be additive when combined.

Aim of this study was therefore to assess the effects of a voluntary versus electrically induced contraction protocol, alone or in combination, in enhancing the different components of functional fitness (muscle strength, cardiopulmonary performance, balance and gait ability) in a group of long-stay hospitalized old women.

\section{Subjects and methods}

\subsection{Subjects}

Long-stay hospitalized old women were screened for eligibility according to the following inclusion criteria: (1) age $\geq 75$ years; (2) hospital stay duration of at least one month; (3) autonomous ambulation; (4) absence of severe cognitive disorders, as assessed by routine clinical tests for this patient population (Mini Mental State Examination, MMSE $>24 / 30$ and Barthel Index $>70 / 100$ ). The MMSE (Folstein et al., 1975) is a 30-item questionnaire assessing cognitive functions. The Barthel Index (Mahoney and Barthel, 1965) measures the level of patient independence in 10 activities of daily living: feeding, transfers, personal self-care, toilet transfers, bathing, walking, stairs climbing, dressing and bladder and bowel continence.

Exclusion criteria were: (1) history of acute stroke; (2) any clinical condition contraindicating mobilization (e.g. deep vein thrombosis, fractures, etc.); (3) physical inability to ambulate or exercise; (4) severe cardiopulmonary diseases; (5) metabolic/ vascular diseases which could impair walking, as diabetes complicated with macroangiopathies, vascular obstructive pathologies causing claudicatio intermittens, etc.

Fifty-seven age-eligible patients were initially screened in a single rehabilitation centre (the Don C. Gnocchi Foundation, Milan, Italy) from January 2006 to July 2007. Forty (age: $82 \pm 7$ years, body mass: $59.5 \pm 12.3 \mathrm{~kg}$, stature: $152 \pm 7 \mathrm{~cm}$, mean \pm S.D.) of these subjects were eligible for inclusion and were randomized (see Fig. 1 for detailed study design). Among the eligible subjects, 19 patients were hospitalized for cardiovascular diseases (arterial hypertension, chronic venous insufficiency, chronic cerebrovascular disease, etc.), 16 for orthopedic pathologies (poliarthroses, osteoporosis, arthritis, etc.) and 5 for neurological diseases (mild cognitive impairment, depressive syndrome, etc.) (see Table 1 for demographic characteristics of enrolled patients). After receiving a full explanation of the purpose of the study and of the experimental procedures, all subjects signed a written informed consent. This study was approved by the ethical committee of the Don C. Gnocchi Foundation and performed according to the principles of the Declaration of Helsinki.

\subsection{Study design}

This study was a randomized, assessor blinded, controlled trial (Fig. 1). All measures were performed by two investigators, who were blinded to the group allocation of participating subjects. Due to the nature of the treatments, patients and therapists were not blinded.

\subsection{Randomization}

A simple randomization procedure, based on a computerized random number generator, was used for treatment allocation. As the effects of the different rehabilitative programs were unlikely to be affected by age and anthropometric features of the patients, randomization was not stratified for these characteristics. Nevertheless, all groups resulted to be matched for age and anthropometric characteristics after randomization (Table 1 ).

Three groups were assigned to 3 different rehabilitation programs (Fig. 1): (1) kinesitherapy applied to lower limbs $(n=10)$, (2) electrical stimulation of lower limbs $(n=10)$ and (3) KT alternated to ES (KT + ES; $n=10$ ). The fourth group did not perform any specific training, and was considered as control (C; $n=10$ ). Each program consisted of 18 training sessions of similar duration (45 min), administered 3 times a week, with at least a day of recovery between two consecutive sessions, for a total duration of 6 weeks. All training sessions were carried out by the same physical therapist.

\subsection{Rehabilitation programs}

The KT program aimed at improving trophism and strength of lower limbs, in particular of leg extensor muscles. Each training session started with a moderate active warm-up, consisting of a 8min cycling exercise at the $50 \%$ of maximal theoretical heart rate, calculated according to Tanaka et al. (2001) equation. According to the ACSM-AHA guidelines for physical activity in older adults (Nelson et al., 2007), the muscle-strengthening exercises included both isotonic and isometric contractions. For isotonic exercises, a load allowing the subject to perform a series of 15-20 repetitions was used. Each isotonic exercise was performed 3 times, with a rest of at least $2 \mathrm{~min}$ between each series. For isometric exercises, contractions were kept for $6-10$ s for $10-15$ repetitions. Each isometric exercise was performed 3 times, with a rest of at least 2 min between each series. Both isotonic and isometric sessions ended with a 10 min passive stretching of lower limb muscles.

The ES program aimed at strengthening the quadriceps muscle and improving its endurance (Caggiano et al., 1994; Laborde et al., 2004). The ES group underwent 18 electrical stimulation sessions of the quadriceps muscle of both legs. Subjects were seated on a chair with the hip and knee placed at $75^{\circ}$ of flexion. The ankle was stabilized with an external support to prevent any isotonic contraction of the quadriceps muscle. An electrical stimulator (mod. Compex 2, Compex, Geneva, Switzerland), delivering compensated, bi-phasic square-waves, was used. Each ES session started with an active warm-up, similarly to the KT protocol. Rectangular cathodes $(10.4 \mathrm{~cm} \times 5.2 \mathrm{~cm})$ were applied on the most proximal motor point of the vastus lateralis, vastus medialis and rectus femoris muscles, whereas a square anode $(5.2 \mathrm{~cm} \times 5.2 \mathrm{~cm})$ was positioned proximally on the thigh. Stimulation amplitude was set at each subject's point of discomfort and was adjusted before each session. Stimulation frequency was increased from $35 \mathrm{~Hz}$ for the first six sessions, to $75 \mathrm{~Hz}$ for the following six ( 7 th to 12th) and to $85 \mathrm{~Hz}$ for the last six (13th to 18th) sessions, according to the manufacturer's instructions. A passive stretching routine similar to the one used for the KT protocol was finally administered.

The KT + ES program consisted of 9 sessions of KT and 9 sessions of ES, alternately administered.

The $C$ group was used as control and underwent routine passive rehabilitative procedures only.

\subsection{Outcome measures}

The four main components of functional fitness, as defined by Nakamura et al. (2007) were used as outcome measures. Each 


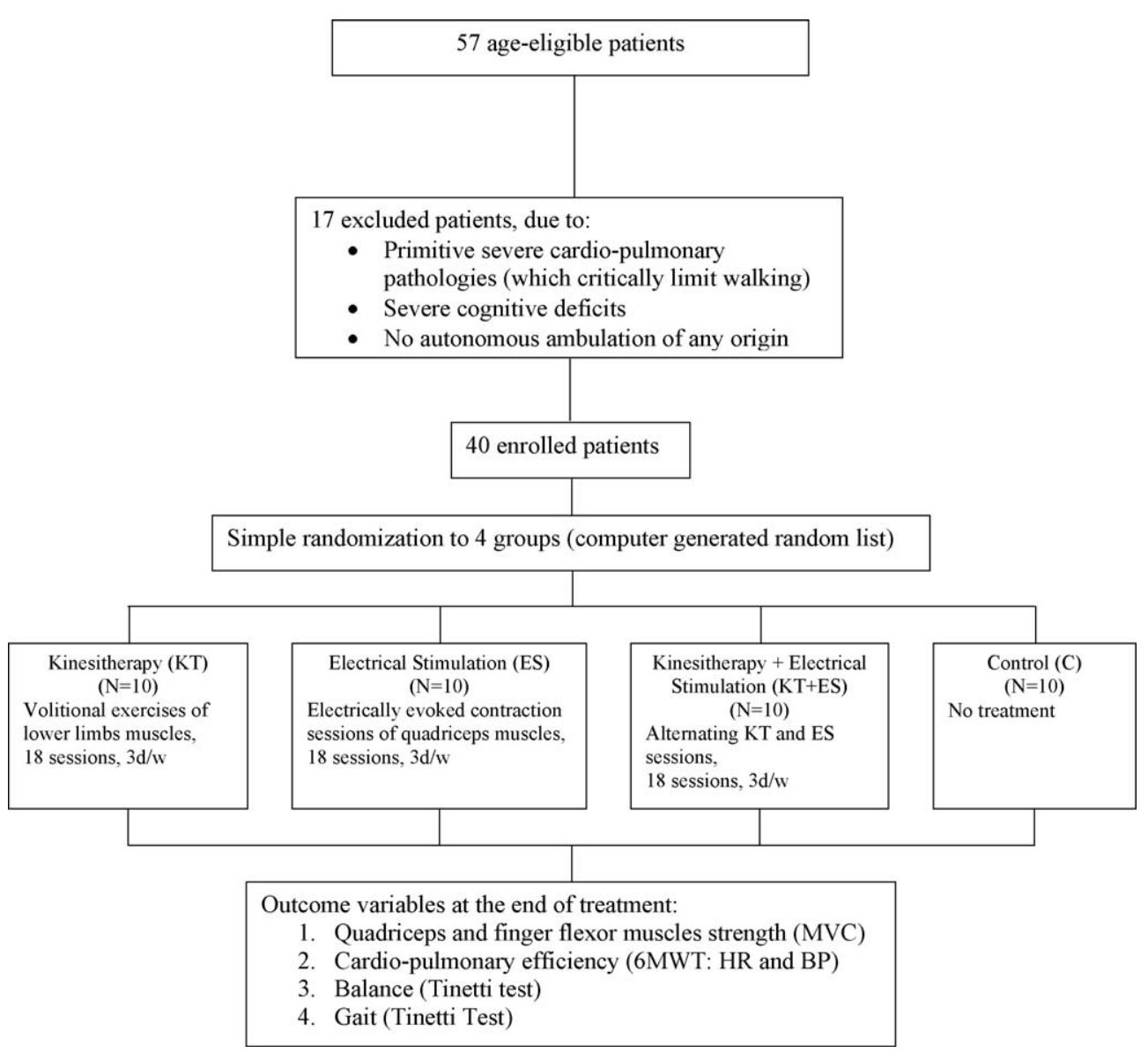

Fig. 1. Study design. $d / w$, days per week.

Table 1

Demographic characteristics of enrolled patients.

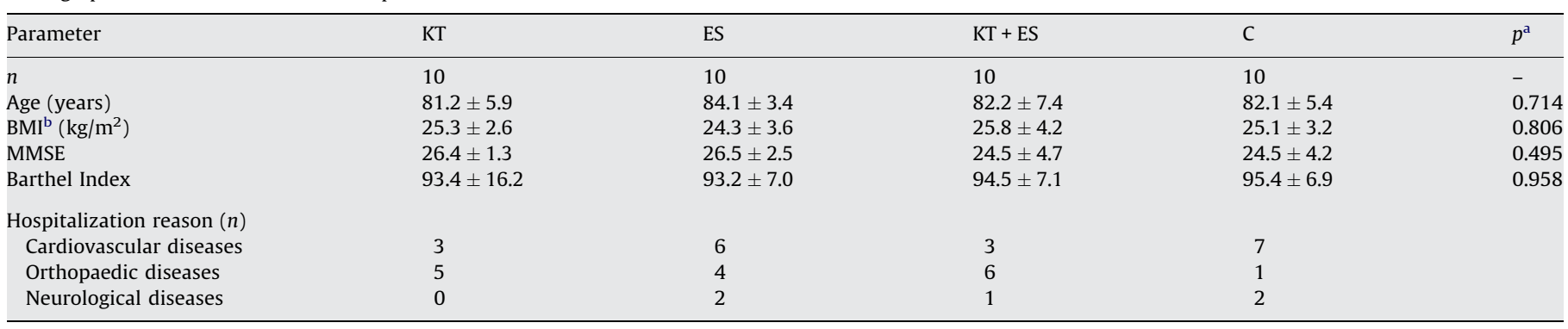

${ }^{a} p$ value for 1-way ANOVA.

b Body Mass Index.

subject was tested at baseline $\left(T_{0}\right)$ and after rehabilitation $\left(T_{1}\right)$, approximately at the same time in the morning. The following parameters were evaluated: maximal isometric lower limb muscle strength, forearm muscle strength, cardiorespiratory fitness and balance and gait skills.

Maximal isometric lower limb muscle strength was assessed by a maximal voluntary contraction (MVC) of the quadriceps femori. Leg extensor muscle MVC was evaluated at a hip and knee angle of both $30^{\circ}$ and $60^{\circ}$ (complete hip and knee extension representing $0^{\circ}$ ). Each subject was placed on an anatomical ergometer with hip, knees and ankles immobilized with an inelastic strap. The output force generated by the leg extensor muscles was measured by a load cell (model SM2000N, Interface, United Kingdom), linearly operating between $0 \mathrm{~N}$ and $2000 \mathrm{~N}$, placed on the ergometer. Each subject was asked to perform 3 MVCs of $3 \mathrm{~s}$ each at both working angles $\left(30^{\circ}\right.$ and $\left.60^{\circ}\right)$. A resting pause of at least 3 min was done between each trial. The best value of the 3 trials was taken as MVC.

Forearm muscle strength was measured by finger flexor MVC. The finger flexor MVC of the dominant upper limb was measured by a mechanical handgrip (Baseline, FEI, Irvington, NY 10533, USA). The subject was sitting on a chair and the arm was positioned straight to the trunk, with the elbow joint set at $90^{\circ}$. Each subject was asked to perform 3 MVCs of $3 \mathrm{~s}$ each, and the best value was taken as MVC.

Cardiorespiratory fitness was evaluated by a 6-min walking test (Enright et al., 2003). Each subject was asked to walk at maximal speed in order to cover the longest possible distance in $6 \mathrm{~min}$. The test was carried out in the local gym of the rehabilitative unit. HR and blood pressure (BP) were measured before and $1 \mathrm{~min}$ and 5 min after the test. A HR monitor (mod. S810, Polar, Finland) with 
a $\mathrm{R}-\mathrm{R}$ resolution and a standard sphygmomanometer (Certus, Tema, Germany) were used.

Balance and gait skills was assessed by Tinetti Balance and Gait test (Performance-Oriented Mobility Assessment, POMA) (Tinetti, 1986). Tinetti Balance and Gait Scale reproduced the position changes, and the balance and gait skills necessary to efficiently and safely execute daily life activity (Gill et al., 1995). Both balance (evaluation range from 0 to 16 ) and gait skills (evaluation range from 0 to 12 ) were measured.

All selected tests were previously checked for reliability in the elderly (Rantanen et al., 1994; Gill et al., 1995; Enright et al., 2003). For our study they were carried out by the same physical therapist, who was blinded to the group allocation of each participant.

\subsection{Statistical analysis}

If not otherwise stated, results are presented as mean \pm stanstandard deviation (S.D.). All parameters were normally distributed (Shapiro-Wilk test) and there were no missing data. Sample size was calculated using the standard deviations obtained from preliminary data. A $10 \%$ difference ( $p=0.05$, two sided) in improving quadriceps isometric strength between groups (with $\beta=0.20$ and $\alpha=0.05$ ) would require a sample of 8 subjects for each group, a $10 \%$ improvement in 6MWT would require 10 subjects for group, and a $10 \%$ difference in Tinetti test balance and walking scores would require 7 subjects for group. Ten patients/group was therefore considered an appropriate sample size to test all hypothesis.

The Student's $t$-test for paired samples was applied to test the difference between $T_{0}$ and $T_{1}$ of the individual variables for each group. In addition, a two-way analysis of variance (2-way ANOVA) with repeated measure was applied to determine the differences between variables and groups at $T_{0}$ and $T_{1}$, with a between-subject factor at 4 levels (4 experimental groups) and a within-subject factor at 2 levels (time: baseline $\left[T_{0}\right]$ and post-treatment $\left[T_{1}\right]$ ). The interaction of group and time provided information on the efficacy of the different treatments on the outcome measures. The post hoc Holm-Sidak test was applied where necessary, to establish the location of the differences. The level of statistical significance was set at $p<0.05$.

Statistical analyses were performed using a commercially available statistical software (SigmaStat for Windows, vers. 3.11, Systat Software Inc., USA).

\section{Results}

All subjects completed the 6-week rehabilitation program without interruptions.

\subsection{Quadriceps MVC}

The MVC values of quadriceps muscles at both working angles $\left(30^{\circ}\right.$, upper panel and $60^{\circ}$, lower panel) after the different rehabilitation programs are shown in Table 2 , as percentage of $T_{0}$. MVC values were normalized for body weight. At $30^{\circ}$, no significant difference was found in $T_{1}$ versus $T_{0}$ after KT, ES and

Table 2

Functional outcome measures.

\begin{tabular}{lrcrr}
\hline & KT (\% of $\left.T_{0}\right)$ & ES (\% of $\left.T_{0}\right)$ & \multicolumn{1}{l}{$\begin{array}{l}\text { KT }+ \text { ES } \\
\left(\% \text { of } T_{0}\right)\end{array}$} & C (\% of $\left.T_{0}\right)$ \\
\hline 6MWT (distance) & $14.9 \pm 6.5^{*}$ & $14.0 \pm 4.6^{*}$ & $9.6 \pm 4.3^{*}$ & $8.1 \pm 4.0$ \\
MVC 30 /BW & $3.4 \pm 7.0$ & $7.8 \pm 5.9$ & $10.5 \pm 7.3$ & $-0.31 \pm 6.8$ \\
MVC 60\%W & $8.2 \pm 6.7$ & $25.8 \pm 11.3^{*}$ & $16.3 \pm 5.2^{*}$ & $4.9 \pm 5.9$ \\
Balance (Tinetti test) & $11.3 \pm 4.6^{*}$ & $9.4 \pm 3.7^{*}$ & $11.3 \pm 3.0^{*}$ & $2.0 \pm 4.6$ \\
Gait (Tinetti test) & $8.2 \pm 4.2$ & $9.8 \pm 6.9$ & $0.8 \pm 3.3$ & $0.9 \pm 3.2$ \\
\hline
\end{tabular}

$p<0.05 T_{1}$ versus $T_{0}$.
$\mathrm{KT}+\mathrm{ES}$. At $60^{\circ}$, MVC significantly increased after ES and KT + ES (from $5.1 \pm 3.3 \mathrm{~N} / \mathrm{kg}$ to $5.6 \pm 1.2 \mathrm{~N} / \mathrm{kg}$ and from $7.0 \pm 0.7 \mathrm{~N} / \mathrm{kg}$ to $8.0 \pm 1.1 \mathrm{~N} / \mathrm{kg}$, respectively, $p<0.05$ for both comparisons). The 2 way ANOVA for repeated measures showed no significant interaction between the two main factors (time $\times$ treatment).

\subsection{Finger Flexor MVC}

The percentage changes in finger flexors MVC of the dominant upper limb were $+2.7 \pm 3.0 \%$ in KT group, $+4.0 \pm 11.1 \%$ in ES group, $+7.9 \pm 6.1$ in KT + ES group, and $-0.5 \pm 3.0 \%$ in C group. No difference was found both inter-groups and between $T_{0}$ and $T_{1}$ in each group. No interaction between the two main factors was found.

\section{3. $6 M W T$}

The percentage changes in the distances covered during the 6 MWT after the different rehabilitation programs are shown in Table 2. Significant differences were found in the distances covered at $T_{1}$ compared to $T_{0}$ after KT, ES and KT + ES (from $227 \pm 80 \mathrm{~m}$ to $249 \pm 77 \mathrm{~m}$, from $225 \pm 66 \mathrm{~m}$ to $255 \pm 70 \mathrm{~m}$ and from $271 \pm 88 \mathrm{~m}$ to $300 \pm 116 \mathrm{~m}$, respectively; $p<0.05$ for all comparisons). No significant interaction between the two main factors was observed. No change in the covered distance was observed in the $C$ group.

Table 3 shows HR values before, immediately after the 6MWT (1st $\mathrm{min}$ ) and after $5 \mathrm{~min}$ of recovery, at $T_{0}$ and $T_{1}$ time points for the different treatment groups. In the KT group, HR significantly decreased at $T_{1}$ compared to $T_{0}$, both before and immediately after the 6MWT $(-4.0 \pm 0.4 \%$ and $-10.1 \pm 1.3 \%$, respectively, $p<0.05)$, and after $5 \mathrm{~min}$ of recovery $(-7.3 \pm 1.4 \%, p<0.05)$. In the $\mathrm{KT}+\mathrm{ES}$ group HR was also significantly lower immediately after the 6MWT $\left(-11.2 \pm 0.8 \%, p<0.05 T_{1}\right.$ vs. $\left.T_{0}\right)$. After the 6MWT, inter-group differences were found in KT and KT + ES with respect to ES and C. No interaction between the two main factors was found.

Systolic and diastolic blood pressure values before and after the 6MWT, measured at $T_{0}$ and $T_{1}$ time points, are shown in Table 3. Neither systolic BP nor diastolic BP significantly changed after any rehabilitation program in any experimental condition.

\subsection{Tinetti test}

The balance and gait skills, as assessed by the Tinetti scale, were separately considered. The balance skill scores (Table 2), significantly increased at $T_{1}$ compared to $T_{0}$, to a similar extent in KT, ES and KT + ES groups (from $12.0 \pm 1.0$ to $13.1 \pm 0.9$, from $11.7 \pm 1.0$ to $12.5 \pm 0.8$ and from $12.8 \pm 0.8$ to $14.1 \pm 0.7$, respectively, $p<0.05$ for all comparisons). No inter-group difference and no interaction between the two main factors were observed.

Conversely, no significant change occurred in the gait skill scores in the different groups (Table 2).

\section{Discussion}

The main findings of this work were the following: (i) the maximal isometric knee extension was significantly improved after ES and KT + ES only; (ii) all treatments similarly increased the distance covered during the 6MWT, but a significant improvement in cardiovascular performance (as assessed by HR recovery) occurred after KT and, to a lesser extent, KT + ES only; (iii) all rehabilitative programs produced a similar effect in static and dynamic balance tasks, but none of them induced a significant improvement of gait skill, as assessed by the Tinetti test.

Quadriceps MVC increased significantly after ES and KT + ES at a joint angle of $60^{\circ}$ only. This can be due to the more selective recruitment of quadriceps muscle by the ES compared to the KT protocol, although at specific contraction angles only. Indeed, 
Table 3

Heart rate and blood pressure before and after 6MWT.

\begin{tabular}{|c|c|c|c|c|c|c|c|c|}
\hline & \multicolumn{2}{|l|}{ KT } & \multicolumn{2}{|l|}{ ES } & \multicolumn{2}{|l|}{$\mathrm{KT}+\mathrm{ES}$} & \multicolumn{2}{|l|}{$\mathrm{C}$} \\
\hline & $T_{0}$ & $T_{1}$ & $T_{0}$ & $T_{1}$ & $T_{0}$ & $T_{1}$ & $T_{0}$ & $T_{1}$ \\
\hline \multicolumn{9}{|l|}{ HR (bpm) } \\
\hline Rest $^{\mathrm{a}}$ & $89 \pm 6$ & $80 \pm 11^{*}$ & $81 \pm 14$ & $81 \pm 15$ & $75 \pm 7$ & $78 \pm 9$ & $75 \pm 16$ & $76 \pm 12$ \\
\hline After $6 \mathrm{MWT} \mathrm{T}^{\mathrm{b}}$ & $104 \pm 15$ & $93 \pm 13^{*}, \#$ & $109 \pm 10$ & $106 \pm 17$ & $94 \pm 15$ & $88 \pm 14^{*}, \#$ & $93 \pm 15$ & $96 \pm 15$ \\
\hline 5-Min recovery & $86 \pm 17$ & $80 \pm 16^{*}$ & $72 \pm 8$ & $76 \pm 14$ & $92 \pm 11$ & $85 \pm 7$ & $80 \pm 17$ & $84 \pm 14$ \\
\hline \multicolumn{9}{|l|}{ Systolic BP (mmHg) } \\
\hline Rest & $130 \pm 15$ & $131 \pm 12$ & $131 \pm 15$ & $131 \pm 14$ & $125 \pm 11$ & $126 \pm 9$ & $134 \pm 11$ & $131 \pm 13$ \\
\hline After 6MWT & $143 \pm 23$ & $137 \pm 15$ & $145 \pm 9$ & $149 \pm 16$ & $135 \pm 12$ & $134 \pm 9$ & $142 \pm 15$ & $139 \pm 12$ \\
\hline \multicolumn{9}{|c|}{ Diastolic BP (mmHg) } \\
\hline Rest & $77 \pm 11$ & $78 \pm 10$ & $77 \pm 9$ & $77 \pm 10$ & $78 \pm 8$ & $79 \pm 7$ & $79 \pm 7$ & $75 \pm 8$ \\
\hline After 6MWT & $82 \pm 15$ & $80 \pm 13$ & $81 \pm 10$ & $82 \pm 9$ & $84 \pm 10$ & $80 \pm 10$ & $81 \pm 5$ & $76 \pm 9$ \\
\hline
\end{tabular}

aefore 6MWT.

b Immediately after 6MWT.

c After 5 min of recovery from 6MWT.

$p<0.05 T_{1}$ versus $T_{0}$

\# $p<0.05$ inter-groups (2-way ANOVA).

previous studies demonstrated that the increase in MVC induced by an ES program is angle-specific (Martin et al., 1993, 1994). From a practical point of view, this result suggests that only some specific joint angles should be used during ES rehabilitative procedures to obtain a clinically relevant improvement. However, normative data on the relationship between force gain and specific joint working angles in this population are still lacking.

As expected, the maximal isometric force did not increase in finger flexor muscles, as no exercise was specifically addressed to increase forearm muscle force. This suggests that circulating factors (as IGF-1, myostatin, etc.) induced by training of specific muscle groups are not relevant in increasing strength and size of untrained muscle, as proposed by previous studies (Walker et al., 2004).

The distance covered during the 6MWT was significantly increased by all rehabilitation protocols. This result is in agreement with other studies, which found an improvement in 6MWT in older women, both after a traditional exercise training (Nakamura et al., 2007) and after ES or isometric contraction training programs (Durmus et al., 2007). Moreover, our results suggest that an increase of gait velocity can be also induced by the combination of isometric and electrically evoked contractions, although in this case the effect seems to be less than additive, as the distance increase obtained with ES and KT alone was about 15\% each, whereas that obtained with ES + KT was lower than $10 \%$.

We also observed that cardiac work (as assessed by HR at rest, immediately after the 6MWT and during recovery) is presumably reduced by KT and, to a lesser extent, by KT + ES, but not by ES alone. This result is in contrast with previous studies. Durmus et al. (2007) found an increase of functional performance, as measured by the WOMAC index, in a group of 50 women (aged $42-75$ years) suffering from knee osteoarthritis undergoing quadriceps electrical stimulation. Neder et al. (2002) showed an increased distance covered in the walking test and some improvement in the cardiopulmonary parameters in a group of 15 elderly subjects ( 6 females) with chronic obstructive pulmonary disease after a 6week ES training program of lower limbs. These improvements were considered to be the result of muscle strengthening and local capillary density increase. A possible explanation of the differences between our data and the above-mentioned studies could be found in the selection of eligible patients. Indeed, both Durmus' and Neder's works were focused on specific and well defined pathologies, whereas our study included a wider range of conditions: the most common cardiopulmonary pathologies (as arterial hypertension, atrial fibrillation, heart failure of NYHA classes I-II, broncopneumopathies, etc.) were not exclusion criteria for our study, unless they precluded walking. In such a heterogeneous population, which however could be more representative of the general elderly female population, ES training seems not to be sufficient to promote an increase of cardiopulmonary performance. However, a larger number of subjects might be necessary to further evaluate this aspect.

In our study, the KT program produced a decrease of HR at rest, after the 6MWT and during recovery. Although such program mainly included isometric and isotonic voluntary contractions of short duration with complete recovery between exercises, the required cardiopulmonary effort might have exerted a cardiovascular training effect, with a consequently significant reduction of cardiac work during walking. This might also be relevant in limiting the cardiovascular risk in this patient population. Indeed, HR recovery from an exercise test is commonly considered as one of the most important predictors of cardiovascular mortality (Nishime et al., 2000). Similarly, although submaximal, the 6MWT and other similar exercise tests demonstrated to detect exercise capacity as determined by maximal cardiopulmonary exercise testing, and the distance walked in 6 min showed a strong, independent association with mortality (Cole et al., 2000; Miyamoto et al., 2000).

In addition, KT exercises of moderate intensity (15-20 repetitions/series) are typically used to improve muscle resistance to fatigue through an amelioration of their peripheral characteristics, although they also promote a slight increase in maximum voluntary isometric force. These changes would result in a better efficiency of lower limb muscles, allowing the patient to walk at higher speed with lower cardiac work, thus improving cardiovascular performance. Overall, our findings showed that when walking is the primary goal of the rehabilitation procedure, KT may be the best choice among the available physical treatment strategies. Conversely, when rehabilitation is mainly aimed at improving strength of a single muscle or muscle group, ES may be a more appropriate option. This could be the case, for example, of a subject with a severe deconditioning of lower limb muscles following a prolonged period of bed rest, or bone fractures of lower limbs. As the effect on cardiovascular performance of the combination of KT and ES is less than additive, the use of this combined protocol with the aim to improve cardiovascular performance may be questionable. However, further studies are necessary to better elucidate this point.

Regarding arterial blood pressure, no changes were apparently induced by the different intervention protocols on both systolic and diastolic pressures (Table 3). Although endurance training is known to improve peripheral and central arteries distensibility 
and endothelial function, a 6-week endurance training did not alter systolic and diastolic blood pressure at rest and during exercise in healthy subjects (Rakobowchuk et al., 2008). However, limited data are available on old female patients, in which arterial distensibility may be compromised.

Finally, balance was significantly improved by all treatments, with a possible additive effect of ES + KT. Therefore, this result confirms that ES and KT, alone or in combination, could reduce the likelihood of accidental falls in old female patients, which is one of the most relevant causes of hospitalization and severe disability in this population. Conversely, gait skills were not significantly improved by any treatment, although a tendency toward an amelioration was observed after KT and ES trainings. The evaluation of walking asymmetries, step variability, and of the central pattern generation of walking was beyond the scope of this study. However, the lack of effect of the different trainings on gait skills could be due to the initial patient selection, favoring the inclusion of subjects with good walking skills at baseline. However, we cannot exclude at present that the training programs used in this study could be able to improve walking skills in a clinically relevant manner.

\subsection{Study limitations}

A potential limitation of this study is the small number of subjects enrolled in each group, which can affect generalizability. Although the number of patients per group satisfies the requirement of sample size calculation, we cannot exclude that larger intervention groups could have generated different results.

A second potential limitation is that we did not evaluated oxygen consumption, as an objective measure of the patients aerobic capacity. The changes in cardiovascular adaptation to exercise have been estimated by the distance covered during the 6MWT and by HR recovery after the 6MWT only. However, some authors demonstrated that the distance walked in a 6MWT strongly correlates with peak oxygen consumption and oxygen pulse determined in the same patients by cardiopulmonary exercise testing (Miyamoto et al., 2000).

Finally, our study design did not include any follow-up. Further studies are therefore necessary to evaluate the long-term duration of the benefits induced by each rehabilitative procedure.

\section{Conclusions}

In our group of long-stay hospitalized older females, lower limb isometric strength was improved only by the training protocols including ES. Conversely, walking ability was improved by all treatments, whereas cardiovascular performance and recovery from exercise was observed with KT only. A less than additive effect on cardiovascular performance, and an additive effect on balance and on quadriceps strength were observed with the combination of ES + KT protocols.

These results may be relevant in rehabilitating old institutionalized patients, especially of female gender, based on individual requirements and specific therapeutic goals.

\section{Conflict of interest statement}

All authors have no actual competing interests to declare.

\section{Role of the funding source}

This work was granted by the Italian Ministry of Health. Project Title: "Ipokinetic female syndrome. Functional electrical stimulation as a preventive tool in young and elderly patients" [Project $n$. E19MOYCY].

\section{CONSORT statement}

This paper was written according to the recommendations of the CONSORT statement.

\section{References}

Caggiano, E., Emrey, T., Shirley, S., Craik, R.L., 1994. Effects of electrical stimulation or voluntary contraction for strengthening the quadriceps femoris muscles in an aged male population. J. Orthop. Sports Phys. Ther. 20, 22-28.

Cole, C.R., Foody, J.M., Blackstone, E.H., Lauer, M.S., 2000. Heart rate recovery after submaximal exercise testing as a predictor of mortality in a cardiovascularly healthy cohort. Ann. Intern. Med. 132, 552-555.

Creditor, M.C., 1993. Hazards of hospitalization of the elderly. Ann. Intern. Med. 118, 219-223.

Durmus, D., Alayli, G., Canturk, F., 2007. Effects of quadriceps electrical stimulation program on clinical parameters in the patients with knee osteoarthritis. Clin. Rheumatol. 26, 674-678.

Enright, P.L., McBurnie, M.A., Bittner, V., Tracy, R.P., McNamara, R., Arnold, A., Newman, A.B., 2003. The 6-min walk test: a quick measure of functional status in elderly adults. Chest 123, 387-398.

Folstein, M.F., Folstein, S.E., McHugh, P.R., 1975. "Mini-mental state" A practical method for grading the cognitive state of patients for the clinician. J. Psychiatr. Res. 12, 189-198.

Gill, T.M., Williams, C.S., Tinetti, M.E., 1995. Assessing risk for the onset of functional dependence among older adults: the role of physical performance. J. Am. Geriatr. Soc. 43, 603-609.

Gillick, M.R., Serrell, N.A., Gillick, L.S., 1982. Adverse consequences of hospitalization in the elderly. Soc. Sci. Med. 16, 1033-1038.

Hirsch, C.H., Sommers, L., Olsen, A., Mullen, L., Winograd, C.H., 1990. The natural history of functional morbidity in hospitalized older patients. J. Am. Geriatr. Soc. 38, 1296-1303.

Inouye, S.K., Wagner, D.R., Acampora, D., Horwitz, R.I., Cooney Jr., L.M., Hurst, L.D., Tinetti, M.E., 1993. A predictive index for functional decline in hospitalized elderly medical patients. J. Gen. Intern. Med. 8, 645-652.

Laborde, A., Rebai, H., Coudeyre, L., Boisgard, S., Eyssette, M., Coudert, J., 2004. Comparison of two electrical stimulation protocols on quadriceps muscle after anterior cruciate ligament surgery Feasability study. Ann. Readapt. Med. Phys. 47, 56-63.

Lazarus, B.A., Murphy, J.B., Coletta, E.M., McQuade, W.H., Culpepper, L., 1991. The provision of physical activity to hospitalized elderly patients. Arch. Intern. Med. $151,2452-2456$.

Mahoney, F.I., Barthel, D.W., 1965. Functional evaluation: the Barthel Index. Md. State Med. J. 14, 61-65.

Martin, L., Cometti, G., Pousson, M., Morlon, B., 1993. Effect of electrical stimulation training on the contractile characteristics of the triceps surae muscle. Eur. J. Appl. Physiol. Occup. Physiol. 67, 457-461.

Martin, L., Cometti, G., Pousson, M., Morlon, B., 1994. The influence of electrostimulation on mechanical and morphological characteristics of the triceps surae. J. Sports Sci. 12, 377-381.

Miyamoto, S., Nagaya, N., Satoh, T., Kyotani, S., Sakamaki, F., Fujita, M., Nakanishi, N., Miyatake, K., 2000. Clinical correlates and prognostic significance of six-minute walk test in patients with primary pulmonary hypertension. Comparison with cardiopulmonary exercise testing. Am. J. Respir. Crit. Care Med. 161, 487-492.

Mysiw, W.J., Jackson, R.D., . Electrical stimulation. In: Braddom, R.L. (Ed.), Physical Medicine and Rehabilitation. Philadelphia, WB Saunders Company pp. 459487.

Nakamura, Y., Tanaka, K., Yabushita, N., Sakai, T., Shigematsu, R., 2007. Effects of exercise frequency on functional fitness in older adult women. Arch. Gerontol. Geriatr. 44, 163-173.

Neder, J.A., Sword, D., Ward, S.A., Mackay, E., Cochrane, L.M., Clark, C.J., 2002. Home based neuromuscular electrical stimulation as a new rehabilitative strategy for severely disabled patients with chronic obstructive pulmonary disease (COPD). Thorax 57, 333-337.

Nelson, M.E., Rejeski, W.J., Blair, S.N., Duncan, P.W., Judge, J.O., King, A.C., Macera, C.A., Castaneda-Sceppa, C., 2007. Physical activity and public health in older adults: recommendation from the American College of Sports Medicine and the American Heart Association. Med. Sci. Sports Exerc. 39, 1435-1445.

Nichols, J., Hitzelberger, L., Sherman, J., Patterson, P., 1995. Effects of resistance training on muscular strength and functional abilities of community dwelling older adult. J. Aging Phys. Act. 3, 238-250.

Nishime, E.O., Cole, C.R., Blackstone, E.H., Pashkow, F.J., Lauer, M.S., 2000. Heart rate recovery and treadmill exercise score as predictors of mortality in patients referred for exercise ECG. J. Am. Med. Assoc. 284, 1392-1398.

Pekindil, Y., Sarikaya, A., Birtane, M., Pekindil, G., Salan, A., 2001. 99mTc-sestamibi muscle scintigraphy to assess the response to neuromuscular electrical stimulation of normal quadriceps femoris muscle. Ann. Nucl. Med. 15, 397-401.

Piva, S.R., Goodnite, E.A., Azuma, K., Woollard, J.D., Goodpaster, B.H., Wasko, M.C. Fitzgerald, G.K., 2007. Neuromuscular electrical stimulation and volitional exercise for individuals with rheumatoid arthritis: a multiple-patient case report. Phys. Ther. 87, 1064-1077.

Quittan, M., Wiesinger, G.F., Sturm, B., Puig, S., Mayr, W., Sochor, A., Paternostro, T. Resch, K.L., Pacher, R., Fialka-Moser, V., 2001. Improvement of thigh muscles by neuromuscular electrical stimulation in patients with refractory heart failure: a 
single-blind, randomized, controlled trial. Am. J. Phys. Med. Rehabil. 80, 206214.

Rakobowchuk, M., Tanguay, S., Burgomaster, K.A., Howarth, K.R., Gibala, M.J., MacDonald, M.J., 2008. Sprint interval and traditional endurance training induce similar improvements in peripheral arterial stiffness and flow-mediated dilation in healthy humans. Am. J. Physiol. Regul. Integr. Comp. Physiol. 295, R236-R242.

Rantanen, T., Era, P., Heikkinen, E., 1994. Maximal isometric strength and mobility among 75-year-old men and women. Age Ageing 23, 132-137.

Tanaka, H., Monahan, K.D., Seals, D.R., 2001. Age-predicted maximal heart rate revisited. J. Am. Coll. Cardiol. 37, 153-156.
Tinetti, M.E., 1986. Performance-oriented assessment of mobility problems in elderly patients. J. Am. Geriatr. Soc. 34, 119-126.

Vuori, I., 1995. Exercise and physical health: musculoskeletal health and functional capabilities. Res. Q. Exerc. Sport 66, 276-285.

Walker, K.S., Kambadur, R., Sharma, M., Smith, H.K., 2004. Resistance training alters plasma myostatin but not IGF-1 in healthy men. Med. Sci. Sports Exerc. 36, 787793.

Warshaw, G.A., Moore, J.T., Friedman, S.W., Currie, C.T., Kennie, D.C., Kane, W.J., Mears, P.A., 1982. Functional disability in the hospitalized elderly. J. Am. Med. Assoc. 248, 847-850. 